Detlef Gaus • Geselligkeit und Gesellige 


\section{Detlef Gaus}

\section{Geselligkeit und Gesellige}

Bildung, Bürgertum und bildungsbürgerliche Kultur um 1800

Verlag J. B. Metzler Stuttgart · Weimar 
Die Veröffentlichug dieser Arbeit wurde durch die Forschungskommission der Universität Lüneburg gefördert.

Die Deutsche Bibliothek - CIP-Einheitsaufnahme

Gaus, Detlef:

Geselligkeit und Gesellige : Bildung, Bürgertum und bildungsbürgerliche Kultur um 1800 / Detlef Gaus. - Stuttgart ; Weimar : Metzler, 1998

Zugl.: Lüneburg, Univ., Diss., 1997

ISBN 978-3-476-45203-0

ISBN 978-3-476-45203-0

ISBN 978-3-476-04297-2 (eBook)

DOI 10.1007/978-3-476-04297-2

Dieses Werk ist einschließlich aller seiner Teile geschützt. Jede Verwertung außerhalb der engen Grenzen des Urheberrechtsgesetzes ist ohne Zustimmung des Verlages unzulässig und strafbar. Das gilt insbesondere für die Vervielfältigungen, Übersetzung, Mikroverfilmungen und Einspeicherung in elektronischen Systemen.

M \& P Schriftenreihe für Wissenschaft und Forschung

(C) 1998 Springer-Verlag GmbH Deutschland

Ursprünglich erschienen bei J.B. Metzlersche Verlagsbuchhandlung und Carl Ernst Poeschel Verlag GmbH in Stuttgart 1998 
Ich glaube, daß ich nicht lebe, um zu gehorchen oder um mich zu zerstreuen, sondern um zu sein und zu werden...

Ich glaube an die Macht des Willens und der Bildung, mich dem Unendlichen wieder anzunähern...

Ich glaube an die unendliche Menschheit...

Schleiermacher 


\section{Danksagung}

Gerne folge ich an dieser Stelle dem guten Brauch, an all' jene zu denken, die die Entstehung dieser Arbeit begleitet und unterstützt haben. Einigen von ihnen sei auch öffentlich gedankt:

Dank an Herrn Prof. Dr. Hartmut Titze, für den es nie eine Frage war, diese Arbeit als Dissertation zu betreuen, und der mich immer ermutigt hat, meine eigenen Wege zu gehen.

Dank an Herrn Prof. Dr. Reinhard Uhle, dessen nie nachlassende fordernde und fördernde Aufmerksamkeit mich hat selber erfahren lassen, was bildende Geselligkeit bedeutet.

Dank an Herrn AD PD Dr. Peter Stein, dem ich viele wichtige Anregungen und hilfreiche Förderung verdanke.

Dank an Herrn AR Dr. Thomas Lehmann, der mir immer und auf jede Weise hilfreich zur Seite stand, wenn ich Unterstützung brauchte.

Dank an Herrn Dipl.-Sozialök. Martin Sendzik und Herrn Dipl.-Sozialpäd. Michael Frommknecht, ohne deren freundschaftlich-hilfreichen Gespräche und deren technische Unterstützung ich viele Schwierigkeiten bei der Fertigstellung dieser Arbeit nicht so gut hätte meistern können.

Dank an die Graduiertenförderung des Landes Niedersachsen, die mir anderthalb Jahre lang das Privileg gegeben hat, mich nur der Geselligkeit zu widmen.

Besonderer Dank aber meinen Eltern, die mir alle Wege eröffnet haben. Ihnen ist dieses Buch gewidmet. 


\section{Vorwort}

Am Anfang dieser Arbeit über die Berliner Salons um 1800 stand die Faszination einer charakteristischen Geselligkeitskultur, eines zivilisierten intellektuellen Milieus. Mit der Faszination einher ging das Wissen um einen Verlust. Jene spezifische Leichtigkeit des Seins, jene Zukunftsfreudigkeit, jene stilvollen Causerien, die in den Salons zusammentrafen, sind im weiteren Verlauf der Geschichte ihrer Heimstatt beraubt worden. An dieses Untergegangene, Verdrängte, Andere zu erinnern, es zu rehabilitieren und der $\mathrm{Zu}$ wendung weiterer Leser zu versichern, war Motivation dafür, die Anstrengung einer solchen Untersuchung auf sich zu nehmen.

Wie aber kann der unvoreingenommene Leser so in den Bann des Gegenstandes geschlagen werden, daß er die sympathetische Themenzuwendung des Autors nachzuvollziehen vermag? Vielleicht hält der Zettelkasten, der dieser Ausarbeitung Voraussetzung war, noch einige Mittel der Verzauberung bereit. Tatsächlich finden sich letzte übriggebliebene Textbeispiele, zu unsystematisch, um in die Untersuchung eingegangen zu sein, die auf literarisch-essayistische Weise vom Besonderen der Salons erzählen. Und gerade weil sie so hart mit jenen ins Gericht gehen, mag ihr rhetorisches Verführungspotential von besonderem Gewicht sein.

So sei Wilhelm Hauff das Wort gegeben. In seinen „Memoiren des Satans" (1823/1961) läßt er den Teufel höchstpersönlich eine Reise nach Berlin unternehmen. Jenem ist Berlin der Ort in deutschen Landen, an dem er sich einer Gemeinde noch sicher sein kann. Ihr Hochamt feiert sie im Tiergarten, denn hier hat eine Lebensart ihre Heimat, deren Grundlagen ein Vierteljahrhundert zuvor von den Salongästen gelegt wurden (S.306):

Ich saß ... an einem schönen Sommerabend im Tiergarten zu Berlin, nicht weit vom Weberschen Zelt; ich betrachtete mir die bunte Welt um mich her und hatte großes Wohlgefallen an ihr... Lust und Leben wie früher zog durch die grünen Bäume, und der Teufel galt wieder was, wie vorzeiten, und war ein geschätzter, angesehener Mann. Ich konnte mich nicht enthalten, einen Gang durch die buntgemischte Gesellschaft zu machen. Die glänzenden Militärs von allen Chargen mit ihren ebenso verschieden chargierten Schönen, die zierlichen Elegants und Elegantinen, die Mütter, die ihre geputzten Töchter zu Markte brachten, die wohlgenährten Räte, mit einem guten Griff der Kassengelder in der Tasche, und Grafen, Barone, Bürger, Studenten, Handwerksburschen, anständige und unanständige Gesellschaft - sie alle um mich her, sie alle auf dem vernünftigsten Wege, mein zu werden! In fröhlicher Stimmung ging ich weiter und weiter, ich wurde immer zufriedener und heiterer. 
Wie der Romantiker erklärt auch der Jungdeutsche Karl Gutzkow in seiner Autobiographie „Rückblicke auf mein Leben” das Herkommen der Berliner Kultur aus dem Geist der Salons. Und da ihm die Verlusterfahrung des Jetztzeitigen noch nicht Realität ist, kann er dem Leser noch voller Sarkasmus jenes auch ihm schon Frühere vorstellen, auf das Hauff anspielt (1857, S.57f.):

Schlegels Lucinde war der Ausdruck dessen, was man sich damals in einer verabredeten Stunde und bei Aufstellung eines wachsamen Kammermädchens nicht mehr versagte... Berlin gab den Ton der laxen Moral an; denn es war damals keineswegs das pietistische, servile und soldatische Berlin von heute, sondern der Sitz einer in der Wollust des Verwesens begriffenen Regierung, der Tummelplatz der Rouerie und Patronage und der große Venusberg leichter und raffinierter Sitten. Das Beispiel des Thrones heiligte jede Ausschweifung. Die Mode war revolutionär und griechisch genial und der Enthusiasmus für Kunst und schöne Literatur kam hinzu, um für die abgeworfene Mosheimische Moral und die kleinen Gewissensbisse Ersatz und genialische Entschuldigung zu bieten. Aus den ersten Briefen des Rahelschen Nachlasses kann man sich ein gutes Bild jener Zeit entwerfen. Die Brust war sehr entblößt, die Kleider orientalisch weit und schwelgerisch geschnitten, einige Aspasien gaben reizende Toilettenstunden, und die jungen Revolutionäre, Catilina Prinz Louis Schmettau an der Spitze, liefen mit excentrischen Redensarten aus einem Boudoir ins andere. Die Literatur war der Faden, an welchen sich allmählich statt der neuesten Recension in den Horen die heiße und begehrliche Leidenschaft aufzog, welche nur einer Leiter bedurfte. Die Literatur war nicht so absolut wie heute, sondern sie mußte bequemen und gewissensleichten Sitten als Entschuldigung dienen.

Bei aller Ablehnung auch hier Bewunderung, neidvoller Blick auf eine Zeit, da Zivilisiertheit noch etwas galt und dem Menschen zu dienen hatte. Gut einhundert Jahre später urteilt Hannah Arendt, die Rahel-Levin-Biographin, in ihrem „Übungen zum politischen Denken” auf ganz ähnliche Weise. Den Mord, der den Rückweg zu den Salons auf immer abschneidet, bereits hinter sich, spielt sie nicht mehr mit literarischen Boshaftigkeiten, sonderen zügelt ihre Gedanken zu kultursoziologischer Strenge. Noch harscher und scheinbar apodiktischer fällt so ihr Urteil aus (61/94, S.278):

Was wir Massenkultur nennen, ist nichts anderes als die Vergesellschaftung der Kultur, die in den Salons begann. Nur daß die Sphäre des Gesellschaftlichen, die sich vorerst der oberen Klassen und Stände bemächtigte, nachgerade alle Schichten erfaßt hat und damit eben zu einem Massenphänomen geworden ist. Aber all die Eigenschaften, welche die Massenpsychologie inzwischen am Massenmenschen entdeckt hat: seine Verlassenheit (und Verlassenheit ist weder Isoliertheit oder Einsamkeit) bei größter 
Anpassungsfähigkeit; seine Erregbarkeit und Haltlosigkeit; seine außerordentliche Konsumfähigkeit (um nicht zu sagen Verfressenheit) bei völliger Unfähigkeit, Qualitäten zu beurteilen oder auch nur zu unterscheiden; vor allem aber sein Egozentrismus und seine verhängnisvolle Weltentfremdung, die er (ebenfalls schon seit Rousseau) als Selbstentfremdung mißversteht all dieses zeigte sich erst einmal in der 'guten Gesellschaft', in der wir es zahlenmäßig nicht mit Massen zu tun haben. Die ersten Massenmenschen, ist man versucht zu sagen, bildeten so wenig eine Masse im Sinne der Quantität, daß sie sich sogar einbilden konnte, eine Elite zu sein.

Und dennoch: bei aller harschen Kritik hat die Vergesellschaftung von Kultur in den Salons für Hannah Arend eine der Anerkennung würdige Qualität, deren Verlust zu beklagen gerechtfertigt ist, die ins Gedächtnis zu rufen die Beschäftigung mit dem Gegenstand lohnt (ebd.):

Dennoch bestehen sehr erhebliche Unterschiede zwischen der letzten Phase der Vergesellschaftung der Kultur in der Massenkultur und der früheren des Bildungsphilistertums. Diese lassen sich vielleicht am besten und kürzesten am Phänomen der Vergnügungsindustrie vergegenwärtigen, gegen die ja bekanntlich gerade der Bildungsphilister und der Kultursnob die allergrößten Bedenken hegen. Der Bildungsphilister ergriff das Kulturelle als Kulturwert, mit dem er sich eine höhere gesellschaftliche Position sichertehöher nämlich, als sie seiner eigenen Meinung nach ihm von Natur oder Geburt zukam. Die Kulturwerte waren also das, was Werte immer sind, nämlich Tauschwerte, und die Entwertung, die dabei beinahe automatisch ins Spiel kam, lag eben darin, daß Kultur benutzt oder mißbraucht wurde für gesellschaftliche Zwecke. Indem die Kulturwerte von Hand zu Hand gingen, griffen sie sich ab und verloren so die allen Kulturtatsachen ursprünglich eigentümliche Fähigkeit, von sich aus zu ergreifen. Aber diese zu Werten denaturierten Kulturdinge wurden nicht verzehrt und konsumiert; auch in ihrer abgegriffensten Form blieben sie weltlich-objektiver Bestand.

Den Implikationen, die die angeführten Zitate beinhalten, soll nun in Verbindung mit weiteren Fragestellungen systematisch nachgegangen werden. Wenn der geneigte Leser anhand der ausgewählten Passagen einen ersten Eindruck davon bekommen hat, wie anregend eine Beschäftigung mit den Berliner Salons um 1800 und ihren Gästen sein kann, mag er es nicht mehr für anstrengend, sondern für lohnend erachten, der Darstellung auf diesem Wege zu folgen. In dieser Hoffnung sei dieses Buch angegangen. 


\section{INHALT}

I. Einleitung 15

1. Literaturlage und Forschungsstand 15

2. Problemstellung 25

II. Geselligkeit. Theoretische Reflexionen 51

1. Einleitung 51

2. Systematisch-historische Begriffsannäherung 51

2.1. Geselligkeit. Soziologische Annäherungen 52

2.1.1. Kooptation und Kooperation $\quad 52$

2.1.2. Schönheit und Lebensnotdurft $\quad 52$

2.1.3. Form und Stoff $\quad 53$

2.1.4. Zusammenfassung 54

2.2. Geselligkeit. Historische Annäherungen 55

2.2.1. Von der Antike bis zum Ende des Mittelalters 56

2.2.2. Renaissance: Die Wiederentdeckung der Anmut 56

2.2.3. Barock: Das Ideal der Klugheit 57

2.2.4. Französische Klassik: Das Ideal der Höflichkeit 58

2.2.5. Bürgerliche Aufklärung: Das Ideal der Offenheit 58

2.2.6. Spätaufklärung: Das Ideal der Urbanität 60

2.2.7. Zusammenfassung 61

3. Geselligkeit als Utopie in Schleiermachers "Versuch einer Theorie des geselligen Betragens" 61

3.1. Publizistischer und diskursiver Hintergrund 62

3.2. Textrekonstruktion 64

3.2.1. Ausgangspunkt der Interpretation 65

3.2.2. Schleiermachers Ausgangsfrage 66

3.2.2.1. Schleiermachers Methode 67

3.2.2.2. Schleiermachers Erkenntnisgewinn 68

3.2.3. Das Entfremdungspotential der Erwerbsarbeit 69

3.2.4. Das Entfremdungspotential der Privatheit 70

3.2.5. Der kulturkritische Zusammenhang 70

3.2.5.1. Schiller 71

3.2.5.2. Schlegel 72

3.2.5.3. Zusammenfassung 73

3.2.6. Schleiermachers Neukonstruktion des Geselligkeitsbegriffes $\quad 74$

3.2.7. Problematisierung I: Natürliche und moralische Tendenz geselliger Kommunikation $\quad 77$

3.2.7.1. Formaler und materialer Aspekt von Kommunikation $\quad 78$ 
3.2.7.2. Materiales Gesetz: Die Kommunizierbarkeit von Ideen und Gefühlen

3.2.7.3. Formelles Gesetz: Traditionsabbrüche und kommunikatives Ethos

3.2.7.4. Zusammenfassung

3.2.8. Problematisierung II: Geselligkeit im engeren Sinne 87

3.2.8.1. Gemeinschaft der Geselligkeit 93

3.2.8.2. Das Wirkliche und das Mögliche 93

3.2.8.3. Zusammenfassung 97

3.2.9. Problematisierung III: Geselligkeit im weiteren Sinne 98

3.2.9.1. Der gesellige Kommunikationsraum 98

3.2.9.2. Salonrealität und liturgisches Geselligkeitsideal $\quad 100$

3.2.9.3. Geselligkeit als Utopie 103

3.3. Die Grenzen der Geselligkeitsutopie 105

3.3.1. Geteilter Erfahrungshorizont und Lebensstil als Voraussetzung gelingender Geselligkeit 106

3.3.2. Die soziale Begrenztheit von Schleiermachers „Versuch“ 109

4. Schluß 112

III. Berliner Salons. Sozialgeschichtliche Rekonstruktionen 115

1. Einleitung 115

2. Salons - Begriffliche Annäherungen 115

2.1. Historische Schlaglichter 116

2.2. Die Berliner Salons der klassischen Periode 120

2.3. Die Salonieren 126

3. Bedingungsgefüge der Berliner Salons um $1800 \quad 145$

3.1. Salons und Gesellschaften $\quad 145$

3.2. Salons und Judentum 151

3.2.1. Historische und ökonomische Schlaglichter 152

3.2.2. Neuorientierungen 159

3.2.3. Das „Ganze Haus" 162

3.2.4. Traditionsabbrüche 166

3.2.5. Zusammenfassung 170

3.3. Salons und Aristokratie 171

3.3.1. Historische und ökonomische Schlaglichter 172

3.3.2. Das Palais 175

3.3.3. Zusammenfassung 178

3.4. Salons und Urbanität 178

3.4.1. Definitorische Abgrenzungen 179

3.4.2. Objektive Potentiale einer Salonentwicklung 182 
3.4.3. Subjektive Verarbeitungen der Großstadtwahrnehmung

3.4.4. Zusammenfassung

4. Berliner Salons um 1800 - Historikerkonstrukt oder historische Realität?

4.1. Historische und ökonomische Schlaglichter

4.2. Infrastruktur und salongeselliger Stil

5. Schluß

1. Einleitung

2. Die empirische Basis

3. Die Salongäste als Gruppe

3. Alter, Generationenzusammenhang und Denkstil 227

3.1. Ergebnisse

3.2. Interpretation

3.2.1. Der Generationenansatz Karl Mannheims

3.2.2. Konstituenten des Generationenzusammenhanges der Salongäste

3.3. Zusammenfassung

4. Verzeitlichung des Salonbegriffs

5. Spezifische Lebens- und Problemlagen 248

5.1. Frauen in den Salons 248

5.2. Juden in den Salons 254

5.3. Zusammenfassung 257

6. Soziale Schichtung in den Salons 258

6.1. Problemstellung 258

6.2. Operationalisierung 259

6.3. Herkunft der Salongäste und deren eigener Status bei Saloneintritt 260

6.3.1. Die "Oberschicht" 261

6.3.2. Die „Mittelschicht“ 266

6.3.3. Interpretation 269

6.5. Akademiker in den Salons 275

6.6. Autoren und Künstler in den Salons 282

6.6.1. Künstlerische Betätigung der Salongäste 283

6.6.2. Interpretation 289

6.6.3. Resümee 297

6.7. Zusammenfassung 298 
V. Berliner Salongeselligkeit. Literarische Reflexe 303

1. Einleitung 303

2. Die Salons in literarischen Zeitzeugnissen 304

2.1. Die Salonieren 304

2.1.1. Die literarischen Zeugnisse 304

2.1.2. Interpretation 313

2.2. Die Salongäste 320

2.2.1. Die literarischen Zeugnisse 320

2.2.2. Interpretation 322

2.3. Zusammenfassung 329

3. Der Salongeschmack 330

3.1. Die mittlere Kommunikationslage 330

3.2. Der mittlere Geschmack 333

3.3. Zusammenfassung 337

4. Die Salongeselligkeit als ästhetische Inszenierung 337

4.1. Die Theatralisierung von Geselligkeit 339

4.1.1. Der Tee 339

4.1.2. Die Miene 342

4.1.3. Der Auftritt 343

4.1.4. Interpretation 349

4.2. Die Theatralisierung von Literatur 351

4.2.1. Die literarischen Zeugnisse 352

4.2.2. Interpretation 357

4.3. Zusammenfassung 359

5. Schluß 359

VI. Schlußwort 363

$\begin{array}{ll}\text { Anhang I } & 375\end{array}$

Anhang II 433

Anhang III 439

Anhang IV 465

$\begin{array}{ll}\text { Literaturverzeichnis } & 481\end{array}$ 